\title{
DISCRETE MELLIN CONVOLUTION WITH DILATION AND ITS APPLICATIONS
}

\section{PAVEL V. PLASCHINSKY}

Belarusian State University Skariny av.4, 220050 Minsk, Belarus

E-mail: paul@mmf.bsu.unibel.by

\section{ABSTRACT}

The analysis of the discrete Mellin convolution is given. A generalization of rezults from $[4,5]$ is presented. Some applications illustrate the efficiency of proposed methods.

\section{MAIN RESULTS}

Let denote by $l_{\nu, p}$ - the Banach space of sequences $a(n)$, such that $a(n) n^{\nu-1 / p} \in l_{p}, \nu \in \mathbf{R}, 1 \leq p \leq \infty$, with norm

$$
\|a(n)\|_{l_{\nu, p}}=\left\|a(n) n^{\nu-1 / p}\right\|_{l_{p}}
$$

$l_{\nu, p}(x)$ - the Banach space of functional sequences $a(n, x), x \in(0, \infty)$, such that $a_{n} \in l_{\nu, p}$, where $a_{n}=\operatorname{ess} \sup _{x}|a(n, x)|$, with norm

$$
\|a(n, x)\|_{l_{\nu, p}(x)}=\left\|a_{n}\right\|_{l_{\nu, p}}
$$

$L_{\nu, p}$ - the Banach space of functions $f(x), x \in(0, \infty)$, such that $f(x) x^{\nu-1 / p} \in$ $L_{p}, \nu \in \mathbf{R}, 1 \leq p \leq \infty$, with norm

$$
\|f(x)\|_{L_{\nu, p}}=\left\|f(x) x^{\nu-1 / p}\right\|_{L_{p}}
$$

Definition 1. Let

$$
h_{\tau}(n, x)=\sum_{k m=n} a(k, x) b\left(m, k^{\tau} x\right)=\sum_{k \mid n} a(n / k, x) b\left(k,(n / k)^{\tau} x\right), n \geq 1
$$


here $\tau \neq 0, k \mid n$ means that $k$ is divisor of $n$. Sequence $h_{\tau}(n, x)=(a * b)_{\tau}(n, x)$ is said to be discrete Mellin convolution of functional sequences $a(n, x)$ and $b(n, x)$ with $\tau$-degree dilation $\left(D M C_{\tau}\right)$.

Under the fixed $a(n, x)$ and $\tau$ the $D M C_{\tau}$ is a linear operator mapping sequence $b(n, x)$ into $h_{\tau}(n, x)$.

Lemma 1. Let $a(n, x) \in l_{\nu+1 / q, 1}(x)$. Then the $D M C_{\tau}$ is a bounded operator in $l_{\nu, p}(x), \nu \in \mathbf{R}, 1 \leq p \leq \infty$, and

$$
\left\|(a * b)_{\tau}(n, x)\right\|_{l_{\nu, p}(x)} \leq\|a(n, x)\|_{l_{\nu+1 / q, 1}(x)}\|b(n, x)\|_{l_{\nu, p}(x)} .
$$

Proof. It is evident that

$$
h_{\tau}(n, x)=\sum_{k \mid n} a(n / k, x) b\left(k,(n / k)^{\tau} x\right)=\sum_{k \mid n} b\left(n / k, k^{\tau} x\right) a(k, x) .
$$

The convolution turns into

$$
h_{\tau}(n, x)=\sum_{k=1}^{\infty} b_{n k}(x, \tau) a(k, x)
$$

where $B(x, \tau)=\left\{b_{n k}(x, \tau)\right\}$ is a $D M C_{\tau}$ - matrix. For $p=\infty$

$$
\begin{gathered}
\left\|h_{\tau}(n, x)\right\|_{l_{\nu, \infty}(x)}=\sup _{n} n^{\nu}\left|h_{n}\right|=\sup _{n}\left(n^{\nu} \operatorname{ess} \sup _{x}\left|\sum_{k=1}^{\infty} b_{n k}(x, \tau) a(k, x)\right|\right) \\
\quad \leq \sup _{n}\left(\left(\frac{n}{k}\right)^{\nu} b_{n k}\right) \sum_{k=1}^{\infty} k^{\nu} a_{k}=\|a(n, x)\|_{l_{\nu+1,1}(x)}\|b(n, x)\|_{l_{\nu, \infty}(x)} .
\end{gathered}
$$

When $1 \leq p<\infty$ the result follows from the generalised Minkovsky inequality (see [1]):

$$
\begin{gathered}
\left\|h_{\tau}(n, x)\right\|_{l_{\nu, p}(x)}=\left(\sum_{n=1}^{\infty}\left(n^{\nu-1 / p} \operatorname{ess}_{x}\left|\sum_{k=1}^{\infty} b_{n k}(x, \tau) a(k, x)\right|\right)^{p}\right)^{1 / p} \\
\leq \sum_{k=1}^{\infty}\left(\sum_{n=1}^{\infty} a_{k}^{p} b_{n k}^{p} n^{\nu p-1}\right)^{1 / p}=\|a(n, x)\|_{l_{\nu+1 / q, 1}(x)}\|b(n, x)\|_{l_{\nu, p}(x)} .
\end{gathered}
$$

Lemma 2. Discrete Mellin convolution (1) is associative. 
Proof. We have the following equalities

$$
\begin{gathered}
((a * b) * c)_{\tau}(n, x)=\sum_{k m=n}(a * b)_{\tau}(k, x) c\left(m, k^{\tau} x\right) \\
=\sum_{k m=n}\left(\sum_{s t=k} a(s, x) b\left(t, s^{\tau} x\right)\right) c\left(m, k^{\tau} x\right)=\sum_{s t m=n} a(s, x) b\left(t, s^{\tau} x\right) c\left(m, s^{\tau} t^{\tau} x\right) \\
=\sum_{s w=n} a(s, x)\left(\sum_{t m=w} b\left(t, s^{\tau} x\right) c\left(m, s^{\tau} t^{\tau} x\right)\right)=\sum_{s w=n} a(s, x)(b * c)_{\tau}\left(w, s^{\tau} x\right) \\
=\left(a *(b * c)_{\tau}\right)_{\tau}(n, x) .
\end{gathered}
$$

Definition 2. A sequence $a^{-1}(n, x)$ is said to be reciprocal to $a(n, x)$ with respectthe to the $D M C_{\tau}$, if almost everywhere on $(0, \infty)$

$$
\left(a * a^{-1}\right)_{\tau}(n, x)=\left(a^{-1} * a\right)_{\tau}(n, x)=\delta_{n}=\left\{\begin{array}{l}
1, n=1 \\
0, n>1
\end{array}\right.
$$

LEMMA 3. If $a_{\mathrm{inf}}=$ essinf $\operatorname{in}_{x}|a(1, x)|>0$, then the reciprocal sequence may be expressed by the recursion relation

$$
\begin{gathered}
a^{-1}(1, x)=\frac{1}{a(1, x)} ; \\
a^{-1}(n, x)=-\frac{1}{a\left(1, n^{\tau} x\right)} \sum_{\substack{k m=n \\
k<n}} a^{-1}(k, x) a\left(m, k^{\tau} x\right) \\
=-\frac{1}{a(1, x)} \sum_{\substack{k m=n \\
m<n}} a(k, x) a^{-1}\left(m, k^{\tau} x\right), n>1,
\end{gathered}
$$

or in the explicit form

$$
\begin{gathered}
a^{-1}(n, x)=\frac{1}{a\left(1, n^{\tau} x\right)} \\
\sum_{\beta \in A_{n}}(-1)^{|\beta|} \sum_{i(\beta)} \frac{a\left(i_{1}, x\right)}{a(1, x)} \frac{a\left(i_{2}, i_{1}^{\tau} x\right)}{a\left(1, i_{1}^{\tau} x\right)} \cdots \frac{a\left(i_{|\beta|}, i_{1}^{\tau} i_{2}^{\tau} \cdots i_{|\beta|-1}^{\tau} x\right)}{a\left(1, i_{1}^{\tau} i_{2}^{\tau} \cdots i_{|\beta|-1}^{\tau} x\right)}
\end{gathered}
$$


where $A_{n}=\left(\beta=\left(\beta_{2}, \beta_{3}, \ldots, \beta_{n}\right), \beta_{k}=0,1,2, \ldots \mid \prod_{k=2}^{n} k^{\beta_{k}}=n, n \geq 1\right), i(\beta)$ is a set of permutations of naturals corresponding to $\beta_{k} \neq 0$ (number $k$ is taken $\beta_{k}$ times), $|\beta|=\sum_{k=1}^{n} \beta_{k}$. As soon as $a(n, x) \equiv a(n)$ the last formula becomes

$$
a^{-1}(n)=\frac{1}{a(1)} \sum_{\beta \in A_{n}}(-1)^{|\beta|} \frac{|\beta| !}{\beta_{2} ! \beta_{3} ! \cdots \beta_{n} !}\left(\frac{a(2)}{a(1)}\right)^{\beta_{2}}\left(\frac{a(3)}{a(1)}\right)^{\beta_{3}} \cdots\left(\frac{a(n)}{a(1)}\right)^{\beta_{n}} .
$$

Proof. Formulae (2) and (3) directly follow from the Definition 1. Formula (4) will be proved by induction. For $n=1$ (4) gives (2). Suppose (4) be true when $n<k$. Then from the formula (3) and from the induction hypothesis for $n=k$ we obtain

$$
\begin{gathered}
a^{-1}(k, x)=-\frac{1}{a\left(1, k^{\tau} x\right)} \sum_{\begin{array}{c}
s t=k \\
s<k
\end{array}} a^{-1}(s, x) a\left(t, s^{\tau} x\right) \\
=-\sum_{\substack{s t=k \\
s<k}}\left[\sum_{\beta \in A_{s}} \frac{(-1)^{|\beta|}}{a\left(1, k^{\tau} x\right)} \sum_{i(\beta)} \frac{a\left(i_{1}, x\right)}{a(1, x)} \cdots \frac{a\left(i_{|\beta|}, i_{1}^{\tau} i_{2}^{\tau} \cdots i_{|\beta|-1}^{\tau} x\right)}{a\left(1, i_{1}^{\tau} i_{2}^{\tau} \cdots i_{|\beta|-1}^{\tau} x\right)}\right] \frac{a\left(t, s^{\tau} x\right)}{a\left(1, s^{\tau} x\right)} \\
=\frac{1}{a\left(1, k^{\tau} x\right)} \sum_{\alpha \in A_{k}}(-1)^{|\alpha|} \sum_{j(\alpha)} \frac{a\left(j_{1}, x\right)}{a(1, x)} \frac{a\left(j_{2}, j_{1}^{\tau} x\right)}{a\left(1, j_{1}^{\tau} x\right)} \cdots \frac{a\left(j_{|\alpha|}, j_{1}^{\tau} j_{2}^{\tau} \cdots j_{|\alpha|-1}^{\tau} x\right)}{a\left(1, j_{1}^{\tau} j_{2}^{\tau} \cdots j_{|\alpha|-1}^{\tau} x\right)}
\end{gathered}
$$

since $A_{k}=\left\{\alpha=\left(\beta_{2}, \ldots, \beta_{t}+1, \ldots, \beta_{k}\right), \beta \in A_{s}, s t=k\right\}$. Thus statement is proved for arbitrary $n$.

ThEOREM 4. The existence of $m$ sequences $a_{\mu}(n, x)$ from $l_{\nu, 1}(x), \mu=1, \ldots, m$, such that

1) $\left(a_{1} * a_{2} * \ldots * a_{m}\right)_{\tau}(n, x)=a(n, x)$;

2) $\left\|a_{\mu}(n, x)\right\|_{l_{\nu, 1}(x)}<\operatorname{essinf}{ }_{x}\left|a_{\mu}(1, x)\right|+e s s \sup _{x}\left|a_{\mu}(1, x)\right|$

$$
=a_{\mu, \text { inf }}+a_{\mu, 1}, \quad \mu=1, \ldots, m
$$

is sufficient for $a^{-1}(n, x)$ belongs to $l_{\nu, 1}(x)$.

Proof. It follows from 2) that the reciprocal sequence $b^{-1}(n, x)$ belongs to $l_{\nu, 1}(x)$ for any sequence $b(n, x)$. In fact

$$
b_{n}^{-1}=\operatorname{ess} \sup _{x}\left|b^{-1}(n, x)\right| \leq \sum_{\beta \in A_{n}} \frac{1}{b_{\mathrm{inf}}^{|\beta|+1}} \frac{|\beta| !}{\beta_{2} ! \beta_{3} ! \cdots \beta_{n} !} b_{2}^{\beta_{2}} b_{3}^{\beta_{3}} \cdots b_{n}^{\beta_{n}}
$$


where $b_{\text {inf }}=\operatorname{ess} \inf _{x}|b(1, x)|$. Then

$$
\begin{aligned}
\sum_{n=1}^{\infty} n^{\nu-1} b_{n}^{-1} & \leq \sum_{n=1}^{\infty} \sum_{\beta \in A_{n}} \frac{1}{b_{\mathrm{inf}}^{|\beta|+1}} \frac{|\beta| !}{\beta_{2} ! \cdots \beta_{n} !} b_{2}^{\beta_{2}} 2^{(\nu-1) \beta_{2}} b_{3}^{\beta_{3}} 3^{(\nu-1) \beta_{3}} \cdots b_{n}^{\beta_{n}} n^{(\nu-1) \beta_{n}} \\
= & \sum_{s=0}^{\infty} \sum_{|\beta|=s} \frac{1}{b_{\mathrm{inf}}^{s+1}} \frac{s !}{\beta_{2} ! \beta_{3} ! \cdots \beta_{k} !} b_{2}^{\beta_{2}} 2^{(\nu-1) \beta_{2}} \cdots b_{k}^{\beta_{k}} k^{(\nu-1) \beta_{k}} \\
= & \frac{1}{b_{\text {inf }}} \sum_{s=0}^{\infty}\left(\frac{1}{b_{\text {inf }}} \sum_{n=2}^{\infty} n^{\nu-1} b_{n}\right)^{s}=\frac{1}{b_{\text {inf }}} \cdot \frac{1}{1-\frac{1}{b_{\text {inf }}} \sum_{n=2}^{\infty} n^{\nu-1} b_{n}} \\
= & \frac{1}{b_{\text {inf }}-\sum_{n=2}^{\infty} n^{\nu-1} b_{n}}=\frac{1}{b_{\text {inf }}+b_{1}-\|b(n, x)\|_{l_{\nu, 1}(x)}}
\end{aligned}
$$

Thus each sequence $a_{\mu}^{-1}(n, x) \in l_{\nu, 1}(x)$. We deduce from Lemmas 1, 2 that $a^{-1}(n, x) \in l_{\nu, 1}(x)$.

The conditions of theorem 4 are best possible, because there are sequences for which these conditions are necessary and sufficient. For example, $a(n, x)=$ $(1, \alpha, 0, \ldots, 0, \ldots)$.

Examine the operator

$$
\left(M_{a, \tau} f\right)(x)=\sum_{n=1}^{\infty} a(n, x) f\left(n^{\tau} x\right), \tau \neq 0, x \in(0, \infty) .
$$

Lemma 5. If $a(n, x) \in l_{1-\tau \nu, 1}(x)$, then $M_{a, \tau}$ (5) is bounded operator in $L_{\nu, p}, \nu \in \mathbf{R}, 1 \leq p \leq \infty$.

Proof of the lemma follows from the generalised Minkovsky inequality.

Theorem 6. Suppose $a(n, x), b(n, x) \in l_{1-\tau \nu, 1}(x)$. For arbitrary function $f(x) \in L_{\nu, p}, \nu \in \mathbf{R}, 1 \leq p \leq \infty$ it is true that

$$
\left(M_{a, \tau} M_{b, \tau}\right) f=M_{h, \tau} f
$$

where $M_{h, \tau}$ is the operator (5) corresponding to $h_{\tau}(n, x)=(a * b)_{\tau}(n, x)$

Proof.

For any function $f(x) \in L_{\nu, p}$

$$
\left(M_{a, \tau}\left(M_{b, \tau} f\right)\right)(x)=\sum_{n=1}^{\infty} a(n, x)\left(M_{b, \tau} f\right)\left(n^{\tau} x\right)
$$




$$
\begin{gathered}
=\sum_{n=1}^{\infty} a(n, x) \sum_{k=1}^{\infty} b\left(k, n^{\tau} x\right) f\left(n^{\tau} k^{\tau} x\right)=|n k=m| \\
=\sum_{m=1}^{\infty}\left(\sum_{n k=m} a(n, x) b\left(k, n^{\tau} x\right)\right) f\left(m^{\tau} x\right)=\left(M_{h, \tau} f\right)(x) .
\end{gathered}
$$

Rearrangement of summands is possible due to Lemmas 1, 5 .

COROLlaRY 1. Under conditions of the Theorem 4 the following formula is true for arbitrary function $f(x) \in L_{\nu, p}, \nu \in \mathbf{R}, 1 \leq p \leq \infty$

$$
M_{a, \tau}^{-1} f=M_{a^{-1}, \tau} f
$$

Here $a^{-1}(n, x)$ is reciprocal sequence to $a(n, x)$ with respect to the $D M C_{\tau}$.

Proof of this corollary follows from Theorems 4,6 .

Let us consider the following integral equations of the Mellin convolution type on $(0, \infty)$ :

$$
\begin{aligned}
& \int_{0}^{\infty} k\left(x, \frac{x}{t}\right) f(t) \frac{d t}{t}=g(x), \\
& \int_{0}^{\infty} k\left(t, \frac{x}{t}\right) f(t) \frac{d t}{t}=g(x), \\
& \int_{0}^{\infty} k(x, x t) f(t) d t=g(x), \\
& \int_{0}^{\infty} k(t, x t) f(t) d t=g(x),
\end{aligned}
$$

Such equations are well-known when $k(u, w) \equiv m(w)$ is a hypergeometric type function, see [1], [2]. We solve (7)-(10) with the kernels of another special type:

$$
k(u, w)=\sum_{n=1}^{\infty} a(n, u) m\left(n^{\tau} w\right), \tau \neq 0, u, w \in(0, \infty)
$$

Here we suppose that solutions of $(7)-(10)$ with $k(u, w) \equiv m(w)$ are known.

Lemma 7. Let $k(w)=$ ess $\sup |k(u, w)| \in L_{\nu, 1}$. The operators from the left parts of (7),(8) [(9),(10)] are bounded ones from $L_{\nu, p}\left[L_{1-\nu, p}\right], \nu \in \mathbf{R}, 1 \leq$ $p \leq \infty$ in $L_{\nu, p}$. 
Lemma 8. If $a(n, x) \in l_{1-\tau \nu, 1}(x), m(x) \in L_{\nu, 1}$, then (11) is satisfied assumption of Lemma $\%$.

Proof of the Lemmas 7, 8, immediately follows from the generalised Minkovsky inequality. In this case rearrangement of summing and integrating is possible due to the analogue of the Fubini theorem [3].

Using the obtained results we can express equations (7)-(10) with kernel (11)in the form

$$
\begin{gathered}
\left(M_{a, \tau} Q f\right)(x)=\sum_{n=1}^{\infty} a(n, x) \int_{0}^{\infty} m\left(\frac{n^{\tau} x}{t}\right) f(t) \frac{d t}{t}=g(x), \\
\left(Q M_{b, \tau} f\right)(x)=\int_{0}^{\infty} m\left(\frac{x}{t}\right)\left(\sum_{n=1}^{\infty} b(n, t) f\left(n^{\tau} t\right)\right) \frac{d t}{t}=g(x), \\
\left(M_{a, \tau} K f\right)(x)=\sum_{n=1}^{\infty} a(n, x) \int_{0}^{\infty} m\left(n^{\tau} x t\right) f(t) d t=g(x), \\
\left(K M_{c,-\tau} f\right)(x)=\int_{0}^{\infty} m(x t)\left(\sum_{n=1}^{\infty} c(n, t) f\left(n^{-\tau} t\right)\right) d t=g(x),
\end{gathered}
$$

where

$$
\begin{gathered}
b(n, x)=a\left(n, n^{\tau} x\right), \quad c(n, x)=a\left(n, n^{-\tau} x\right) n^{-\tau} \\
(K f)(x)=\int_{0}^{\infty} m(x t) f(t) d t, \quad(Q f)(x)=\int_{0}^{\infty} m\left(\frac{x}{t}\right) f(t) \frac{d t}{t} .
\end{gathered}
$$

All series in (12)-(15) converge in mean under the Lemmas' 7, 8 conditions. With Theorems 4, 6 and formula (6) we obtain the solutions of equations in the following form:

$$
\begin{gathered}
f(x)=\left(Q^{-1} M_{a^{-1}, \tau} g\right)(x), \\
f(x)=\left(M_{b^{-1}, \tau} Q^{-1} g\right)(x), \\
f(x)=\left(K^{-1} M_{a^{-1}, \tau} g\right)(x), \\
f(x)=\left(M_{c^{-1},-\tau} K^{-1} g\right)(x) .
\end{gathered}
$$

This paper generalize the results of [4], [5]. 


\section{REFERENCES}

[1] Samko S.G., Kilbas A.A., Marichev O. Fractional integrals and derivatives. Theory and applications. Gordon and Breach, New York, Philadelphia, London, Paris, Montreux, Tokyo, Melbourne, 1993.

[2] S.B.Yakubovich, Yu.F.Luchko. The Hypergeometric Approach to Integral Transforms and Convolutions. (Math. and its Appl. 287), Kluwers Academic Publishers, Dordrecht, 1994.

[3] Titchmarsh E.C. The Theory of Functions. Oxford, 1932.

[4] Vasil'ev I.L. and Plaschinsky P.V. // Doklady AN Belarusi, 40, No 2, (1966), P. 46-48 (in Russian).

[5] Plaschinsky P.V. // Boundary value problems, special functions and fractional calculus. Proc. of Intern. Conf. dedicated to the 90th birthday of academician F.D. Gakhov (Belarus, Minsk, February 16-20, 1996). Minsk, (1996), P. 279-283 (in Russian). 